\title{
NUEVOS REGISTROS DE HONGOS AGARICALES (BASIDIOMYCOTA) PARA LAS YUNGAS BOLIVIANAS
}

\author{
Elizabeth Melgarejo-Estrada ${ }^{1,2,3,4}$, M. Eugenia Suárez ${ }^{2,3}$ \& Bernardo E. Lechner ${ }^{1,3}$ \\ ${ }^{1}$ Laboratorio de Hongos Agaricales, Departamento de Biodiversidad y Biología Experimental, Facultad de Ciencias \\ Exactas y Naturales, Universidad de Buenos Aires, Intendente Güiraldes 2160, C1428EGA Buenos Aires, Argentina; \\ blechner@bg.fcen.uba.ar (autor corresponsal). \\ ${ }^{2}$ Grupo de Etnobiología, Departamento de Biodiversidad y Biología Experimental, Facultad de Ciencias Exactas y \\ Naturales, Universidad de Buenos Aires, Intendente Güiraldes 2160, C1428EGA Buenos Aires, Argentina. \\ ${ }^{3}$ Instituto de Micología y Botánica (UBA-CONICET), Facultad de Ciencias Exactas y Naturales, Universidad de \\ Buenos Aires, Intendente Güiraldes 2160, C1428EGA Buenos Aires, Argentina. \\ ${ }^{4}$ Herbario del Oriente Boliviano (USZ), Museo de Historia Natural Noel Kempff Mercado, Universidad Autónoma \\ Gabriel René Moreno, Av. Irala 565, Santa Cruz, Bolivia.
}

\begin{abstract}
Melgarejo-Estrada, E; M. E. Suárez \& B. E. Lechner. 2020. New records of Agaric fungi (Basidiomycota) for the Bolivian Yungas. Darwiniana, nueva serie 8(1): 309-317.

Three agaric species are cited for the first time for the Bolivian Yungas mycobiota: Agrocybe perfecta, Hygrocybe atrosquamosa and Mycena holoporphyra. These species are described, illustrated, and data on their distribution and ecology are provided.
\end{abstract}

Keywords. Agaricomycetes; Carrasco National Park; cloud forests; Cochabamba; fungal diversity; humic mushrooms.

Resumen. Melgarejo-Estrada, E; M. E. Suárez \& B. E. Lechner. 2020. Nuevos registros de hongos Agaricales (Basidiomycota) para las Yungas bolivianas. Darwiniana, nueva serie 8(1): 309-317.

Se citan por primera vez tres especies de hongos Agaricales para la micobiota de las Yungas de Bolivia: Agrocybe perfecta, Hygrocybe atrosquamosa y Mycena holoporphyra. Se describen e ilustran estas especies y se proporcionan datos sobre su distribución y ecología.

Palabras clave. Agaricomycetes; bosques nublados; Cochabamba; diversidad de hongos; hongos humícolas; Parque Nacional Carrasco.

\section{INTRODUCCIÓN}

Las "Yungas tropicales" se distribuyen desde el norte de Perú hasta el noroeste de la Argentina (entre los $19^{\circ}$ y $29^{\circ} \mathrm{S}$ de latitud) atravesando Bolivia desde el noroeste hacia el sur. La vegetación característica son los bosques nublados y en Bolivia ocupan una superficie total aproximada de $56.000 \mathrm{~km}^{2}$ (Cabrera \& Willink, 1980; Josse et al., 2011). En el contexto de Bolivia, la provincia biogeográfica de las Yungas Peruano-Bolivianas representa una de las regiones más diversas del país (Kessler \& Beck, 2001) y un importante centro de endemismos en lo que a flora vascular (Navarro \& Maldonado, 2011) y fauna
(Young, 2007) se refiere. Esta provincia se distribuye entre los 2900-3100 m s.m. y los 4000-4200 m s.m., abarcando bioclimas pluviales, pluviestacionales y excepcionalmente xéricos, con diferentes pisos de vegetación muy heterogéneos (Navarro, 2015). Geopolíticamente, las Yungas se encuentran bien representadas en regiones de los departamentos de Cochabamba, La Paz y Santa Cruz (Ibisch \& Mérida, 2003; Navarro \& Maldonado, 2011; Navarro, 2015), principalmente en las faldas orientales de Los Andes, donde se extienden áreas protegidas representativas de esta ecorregión como el Parque Nacional Carrasco, el Parque Nacional Madidi y el Parque Nacional Amboró, respectivamente (SERNAP, 2020). 
En el Parque Nacional Carrasco, que ocupa aproximadamente 622.600 ha (SERNAP, 2020), los estudios realizados hasta el momento se han enfocado en vertebrados y plantas vasculares (Ibisch, 1996; Ibisch et al., 2001), mientras el conocimiento micológico es muy escaso (Piepenbring, 2003; Rocabado et al., 2007; Melgarejo, 2015). Los hongos cumplen un rol importante en el ecosistema como descomponedores de la materia orgánica (Piepenbring, 2015); además, intervienen en los ciclos biogeoquímicos, existiendo numerosos factores edáficos y ambientales que pueden afectar la abundancia y riqueza de diferentes tipos de hongos del suelo (Marín, 2018), incluyendo plantas vasculares.

Hasta el presente fueron documentadas 365 especies de hongos Agaricales para Bolivia (Melgarejo-Estrada et al., 2020a; Melgarejo-Estrada et al., 2020b), de las cuales aproximadamente 142 ocurren en la provincia biogeográfica de las Yungas Peruano-Bolivianas y sólo cuatro fueron reportadas para el Parque Nacional Carrasco: Hygrocybe siparia (Berk.) Singer, Hygrophorus prasinus Berk. \& Broome (Farr \& Stevenson, 1963; MelgarejoEstrada et al., 2020b), Marasmius albogriseus (Peck) Singer y Moniliophthora perniciosa (Stahel) Aime \& Phillips-Mora (Stevenson \& Cárdenas, 1949). El objetivo de este trabajo es reportar tres nuevos registros de especies de hongos Agaricales para el sector centro-sur de las Yungas PeruanoBolivianas, las Yungas del Ichilo, en Bolivia, y proveer una descripción detallada e ilustraciones, así como información sobre su distribución y ecología.

\section{MATERIALES Y MÉTODOS}

Las especies fueron coleccionadas durante la época lluviosa (octubre - marzo) de 2018 y 2019 en dos localidades de las Yungas del Ichilo (Fig.1): Parque Nacional Carrasco $\left(17^{\circ} 23^{\prime} 00^{\prime \prime} \mathrm{S} 65^{\circ} 03^{\prime} 00^{\prime \prime} \mathrm{O}\right.$, 500 m s.m), localizado en el municipio de Chapare; Sehuencas y Santa Isabel (17 11'37’S, 65 51'55”O, 2406 m s.m), localizado en el municipio de Colomi.

Los ejemplares fueron analizados macro- $\mathrm{y}$ microscópicamente siguiendo protocolos estándar para la descripción de hongos Agaricales (Largent, 1986; Singer, 1986) y los conceptos taxonómicos de Lodge et al. (2004), Pegler (1983), Singer \& Digilio (1952) y Niveiro et al. (2011).

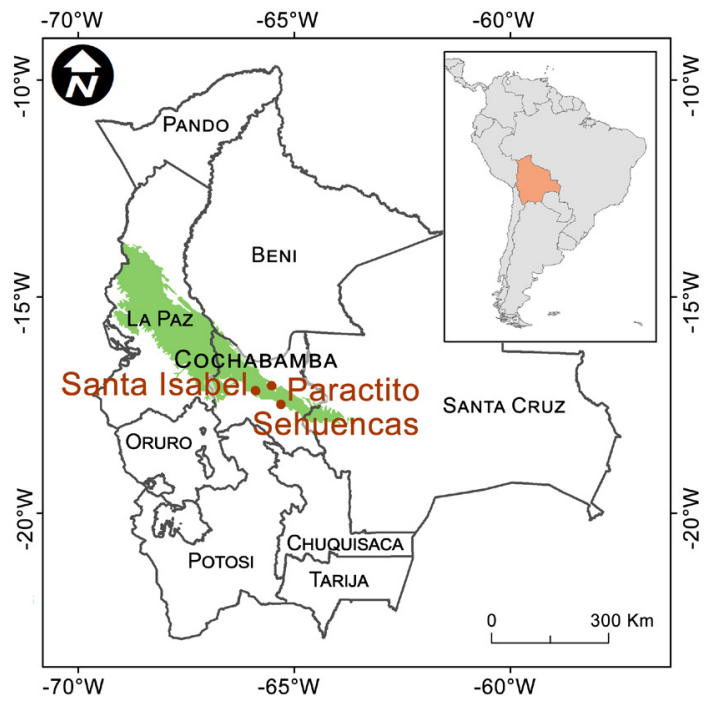

Fig. 1. Región de las Yungas Peruano-Bolivianas (verde). Los puntos indican los sitios en donde se realizaron las colecciones. Figura en color en la versión en línea http:// www.ojs.darwin.edu.ar/index.php/darwiniana/article/ view/890/1188

Para las observaciones microscópicas se realizaron cortes a mano alzada de basidiomas rehidratados, montados en $\mathrm{KOH}$ al $5 \%$ y teñidos con floxina acuosa al 1\%. Se utilizó reactivo de Melzer para verificar la reacción en las paredes de las esporas y en la trama himenoforal (Wright \& Albertó, 2002). Los cortes fueron observados al microscopio óptico con una resolución de 1000x.

En las descripciones se utilizan las siguientes abreviaturas: $\mathrm{N}=$ número de mediciones de basidiosporas; $\mathrm{L}$ y $\mathrm{A}=$ promedio del largo y ancho de las basidiosporas, respectivamente; $\mathrm{Q}=$ cociente calculado entre el largo y el ancho, y $\mathrm{Qm}=$ media del valor de Q ( \pm un desvío estándar). Para indicar los colores se utiliza la terminología propuesta por Kornerup \& Wanscher (1978). Los autores de los nombres científicos se indican de acuerdo con Index Fungorum - Authors of Fungal Names (CABI Bioscience \& Landcare Research, 2020). Los especímenes fueron preservados y depositados en el Herbario del Oriente Boliviano (USZ) en Santa Cruz de la Sierra, Bolivia, y en el Fungario de Buenos Aires Facultad de Ciencias (BAFC), Buenos Aires, Argentina (Thiers 2020, permanentemente actualizado). 


\section{RESULTADOS}

Agrocybe perfecta (Rick) Singer, Lilloa 25:323. 1952 (1951). Pholiota platensis var. perfecta Rick Brotéria, série botanica 6(2): 78 (1907). Pholiota vermiflua var. perfecta Rick, Lilloa 3: 402 (1938), TIPO: Brasil Rio Grande do Sul (Holotipo, en Brotéria, série botanica 6(2): 78, table V. 1907, visto). Figs. 2-3.

Píleo (Fig. 2) 35-65 mm de diám., convexoaplanado, cóncavo al madurar, blanco (1A1) a amarillo pálido (43A), amarillo grisáceo (4B4) en la porción central, blanco amarillento (3A2) en el margen, carnoso, víscido, glabro, levemente arrugada en el centro, margen estriado, translúcido, ligeramente incurvado. Laminillas libres a adnexas, próximas, $3 \mathrm{~mm}$ de diám., castaño claras (6D3), lisas, enteras y ventricosas. Lamélulas de tercer orden, próximas, $3 \mathrm{~mm}$ de ancho, castaño claras (6D3), margen erodado. Pie 95-100 × 20-30 mm, central, cilíndrico, recto, ensanchándose hacia la base, champagne (4B3); crema (4A3) hacia la base, fibrilloso, seco, hueco, superficie subglabra, rizomorfo blanco (5A1). Anillo 15-20 mm de diám., súpero, blanco amarillento (4A2), membranoso, bien desarrollado, persistente y sobresaliente. Olor y gusto no distinguible. Esporada castaño clara.

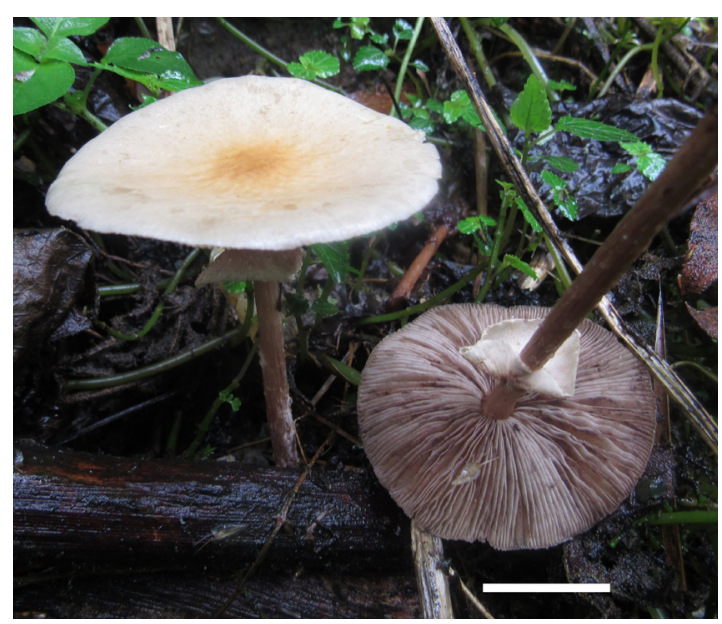

Fig. 2. Aspecto general y hábitat de Agrocybe perfecta (E. Melgarejo-Estrada et al. 382; BAFC, USZ). Escala: $2 \mathrm{~cm}$. Figura en color en la versión en línea http://www.ojs.darwin.edu.ar/index.php/darwiniana/ article/view/890/1188
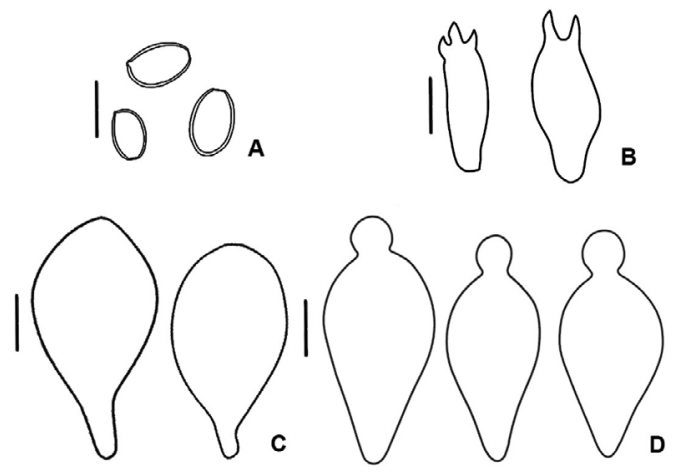

Fig. 3. Agrocybe perfecta. A, esporas. B, basidios. C, pleurocistidios. D, queilocistidios. Escala: $10 \mu \mathrm{m}$.

Esporas (Fig. 3A) 10-12 (-14) $\times$ 5-7 (-9) $\mu \mathrm{m}$, $\mathrm{L}=10,75 \mu \mathrm{m}, \mathrm{A}=5,67 \mu \mathrm{m}, \mathrm{Q}=1,5-2, \mathrm{Qm}=1,91$ $\pm 0,2, \mathrm{~N}=15$, elipsoidales a oblongas, pigmento castaño claro, lisas, algunas con pared gruesa, poro germinativo truncado, inamiloides. Basidios (Fig. 3B) 20-27 (-32) × 7-9 $\mu \mathrm{m}$, cilíndricos, fusiformes, frecuentemente 4-esporados, algunos 2-esporados, pared delgada y hialinos. Pleurocistidios (Fig. 3C) $35-45 \times 20-24 \mu \mathrm{m}$, vesiculosos a piriformes, pared delgada, lisos y hialinos. Queilocistidios (Fig. 3D) 36-45 × 18-21 $\mu \mathrm{m}$, vesiculosos, subcapitados, pared delgada, lisos y hialinos, Trama himenoforal regular, hifas 4-6 $\mu \mathrm{m}$ de diám., hialinas, algunas con pigmento castaño claro. Pileipellis himeniforme, con células vesiculosas a claviformes de 20-37 × 17-24 $\mu \mathrm{m}$. Fíbulas presentes.

Distribución y hábitat. Gregario, creciendo sobre suelo con hojarasca. Agrocybe perfecta es una especie con presencia en Sud América (Coimbra, 2015). Su distribución es conocida para la Argentina en la provincia de Tucumán, Mendoza (Niveiro \& Albertó, 2012; Uhart, 2006) y Salta (Niveiro et al., 2020) y para Brasil (Coimbra, 2015; de Meijer, 2006; Watling, 1992) en el estado de Paraná, creciendo sobre estiércol o suelo con hojarasca. Se reporta por primera vez la presencia de esta especie para el departamento de Cochabamba, en las Yungas del Ichilo de Bolivia.

Observaciones. Agrocybe perfecta se caracteriza por poseer basidiomas robustos, blanquecinos, píleo amarillo grisáceo en la parte central, a veces rugoso, 
aclarándose hacia los márgenes, o amarillento, liso, a veces víscido, involuto en la madurez y de esporada castaño oscura (Singer \& Digilio, 1952; Watling, 1992). Agrocybe perfecta generalmente se encuentra creciendo en humus; sin embargo, Singer \& Digilio (1952) encontraron basidiomas creciendo sobre suelo en un bosque lluvioso de alta montaña en la Argentina, tal como nuestra colección de Bolivia.

Esta especie es muy similar a Agrocybe platensis (Speg.) Singer. Difiere de ella macroscópicamente por poseer el píleo levemente umbonado y rugoso, a veces higrófano en la madurez y laminillas libres a adnexas (Singer \& Digilio, 1952; Watling 1992), mientras que $A$. platensis posee píleo a veces umbonado, liso, no higrófano en la madurez, laminillas adnatas (Singer \& Digilio, 1952; Uhart, 2006). Respecto a la microscopía, las esporas de $A$. platensis son levemente más grandes, 10-14,5 (-16) $\times 7-9,5 \mu \mathrm{m}$, pero superpuestas con A. perfecta; poseen basidios 4-esporados y pleurocistidios vesiculosos con un mucrón o raramente dos, mientras que $A$. perfecta posee basidios 4- y 2-esporados, estos últimos en menor frecuencia, y pleurocistidios vesiculosos sin mucrón (Singer \& Digilio, 1952). En la muestra coleccionada en Bolivia, los queilocistidios encontrados son mucho más grandes $(35-45 \times 20-24 \mu \mathrm{m}$ vs. $17-48 \times 8-30$ $\mu \mathrm{m})$ que los reportados por Singer \& Digilio (1952) y, si bien el basidioma se encuentra en el rango de tamaño, es notablemente más grande que el ejemplar coleccionado por Singer 3344 (BAFC 31892) y similar a los ejemplares esbeltos de la fotografía tipo para $A$. perfecta (Rick, 1907).

A partir de Pholiota vermiflua var. perfecta Rick, Singer \& Digilio (1952) propusieron una combinación nueva: A. perfecta (Rick) Singer, incluyendo las formas levis (basado en Singer T 1326, LIL) y angustisperma (basado en Singer $\mathrm{T}$ 1121, LIL), las cuales son inválidas por no incluir una descripción en latín (Art. 39, Turland et al., 2018). Fue reconocida y explicitada como combinación nueva en Singer (1953), quien al mismo tiempo nombró como sinónimos a Pholiota platensis var. perfecta Rick, y Pholiota vermiflua var. perfecta Rick. Años después, Watling (1992) estudió colecciones de Brasil, y Niveiro et al. (2020) estudiaron el género en la Argentina, aceptando las combinaciones realizadas por Singer \& Digilio (1952) y Singer (1953), quienes diferenciaron $A$. perfecta de A. platensis. En este estudio, y basándonos en los caracteres morfológicos descritos en la literatura para ambas especies (Spegazzini, 1899; Rick, 1938; Singer \& Digilio, 1952; Watling, 1992; Uhart, 2006; Niveiro et al., 2020), consideramos que los caracteres diferenciales descritos anteriormente (basidios 2-4 esporados y pleurocistidios con o sin mucrón), sumado a la superposición de medidas de caracteres importantes (ej. tamaño de esporas y basidios) no son suficientes para separar ambas especies, por lo que se requiere realizar estudios adicionales a nivel molecular y estudios filogenéticos con las especies cercanas, ya que los límites entre ambas especies no son del todo claros, concordando con lo expresado por Niveiro et al. (2020).

\section{Material examinado}

ARGENTINA. Tucumán. Capital, 17-V1959, R. Singer 3344 (BAFC 31892). BOLIVIA. Cochabamba. Colomi, comunidad de Santa Isabel, Pampa Tambo, camino hacia el río Vinto, sobre suelo, 6-X-2019, E. Melgarejo-Estrada et al. 382 (BAFC 553394, USZ), $17^{\circ} 11^{\prime} 44^{\prime \prime} \mathrm{S}, 65^{\circ}$ 52' 56" O, $2398 \mathrm{~m}$ s.m.

Hygrocybe atrosquamosa Pegler, Kew Bulletin Additional Series 9: 53. 1983. TIPO: Martinica, Fiard 932A. (Holotipo K, no visto). Figs. 4-5.

Píleo (Fig. 4) 35-40 mm de diám., aplanado, cóncavo cuando maduro, con umbón, rosado (8A2)a anaranjado rojizo (8A7) cuando adulto, amarillento (5A6) cuando joven, delgado, higrófano, glabro, margen rimoso cuando adulto. Laminillas libres, subdistantes a distantes, 4-5 $\mathrm{mm}$ de diám., a veces naranja amarillentas (6A7) en la base, rojo pastel (8A4), naranja pálido (6A3) a blanco (6A1) hacía la arista, erodadas. Lamélulas de tercer orden, subdistantes a distantes, $4 \mathrm{~mm}$ de diám., blancas (6A1), erodadas. Pie 30-40 × 5-5,5 mm, central, cilíndrico, recto, amarillo (5A6) a rojo pálido (8A3) cuando joven, amarillo rojizo (8A7) cuando maduro, casi transparente, fibrilloso, húmedo, hueco, glabro, desprendiendo un líquido claro al corte, no muy abundante. Anillo ausente. Olor y gusto no distinguible. Esporada blanca. 

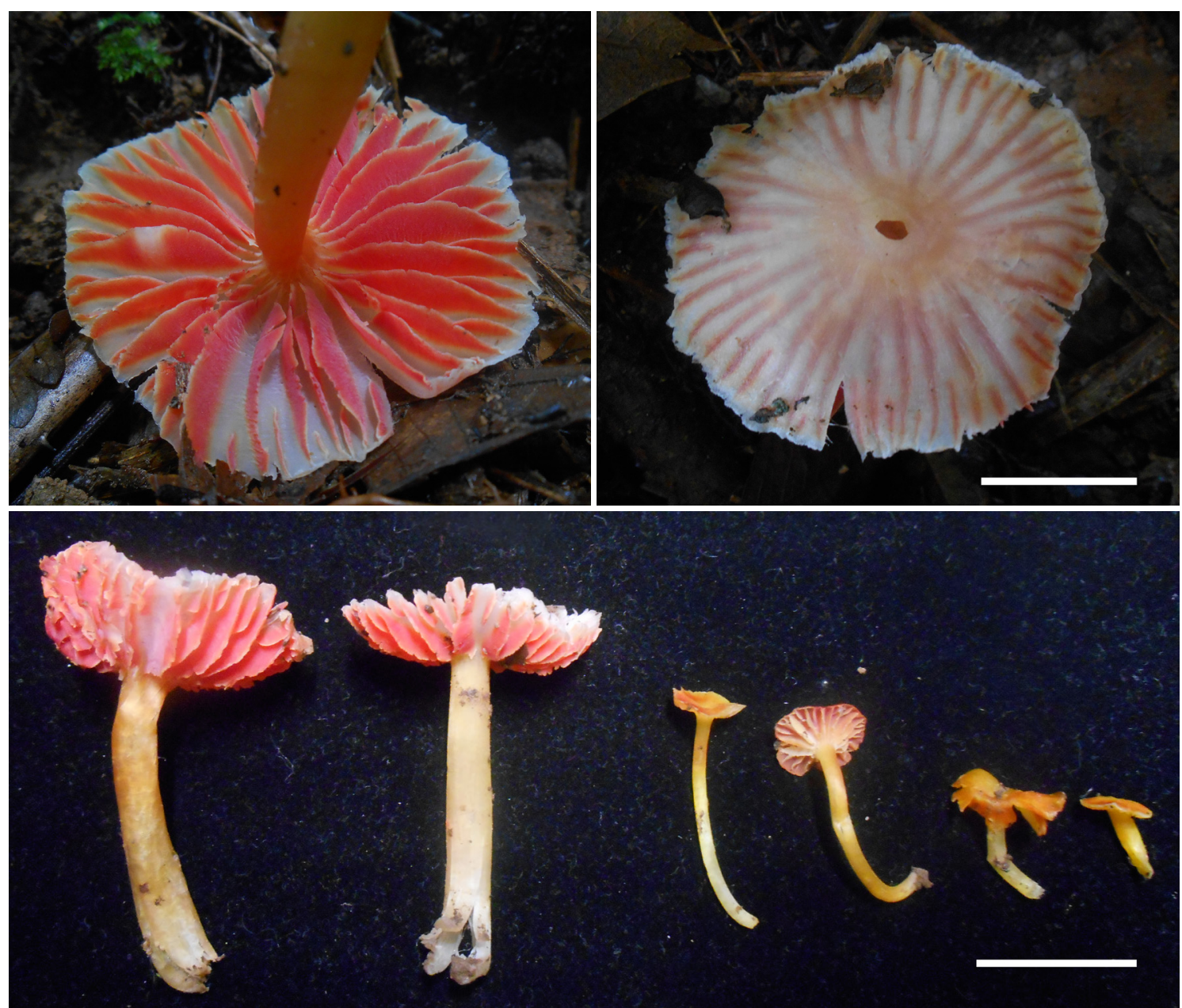

Fig. 4. Aspecto general de Hygrocybe atrosquamosa (E. Melgarejo-Estrada \& M. Ibarra-Merida. 128; BAFC). Escala: $2 \mathrm{~cm}$. Figura en color en la versión en línea http://www.ojs.darwin.edu.ar/index.php/darwiniana/article/view/890/1188

Esporas (Fig. 5A) 7-9 $(-10) \times 3-6 \mu \mathrm{m}$, $\mathrm{L}=8,64 \mu \mathrm{m}, \mathrm{A}=4,50 \mu \mathrm{m}, \mathrm{Q}=1,5-1,9, \mathrm{Qm}=1,8$ $\pm 0,35, \mathrm{~N}=24$, subglobosas a ovoides, hialinas, lisas, pared delgada, gutuladas, inamiloides. Basidios (Fig. 5B) 30-45 × 6-7 (-10) $\mu \mathrm{m}, 2$ a 4-esporados, claviformes, de pared delgada y hialinos; esterigmas de hasta de $6 \mu \mathrm{m}$ de largo. Pseudocistidios (Fig. 5C) 30-55 × 4-7 $\mu \mathrm{m}$, fusoides, a veces apretados hacia el ápice, pared delgada, hialinos. Queilocistidios ausentes. Trama himenoforal regular, hifas $10-20 \mu \mathrm{m}$ de diám., hialinas, algunas con contenido refractivo. Pileipellis cutis bien diferenciado, con hifas repentes, pared lisa y delgada. Fíbulas ausentes.
Distribución y hábitat. Gregario, creciendo sobre suelo. Hygrocybe atrosquamosa es una especie con presencia en India (Farook et al., 2013) y el Neotrópico, encontrándose en Brasil (Lodge \& Pegler, 1990), Martinica (Pegler, 1983), México (Lodge \& Pegler, 1990), Puerto Rico (Lodge \& Pegler, 1990), República Dominicana (Cantrell \& Lodge, 2000) y Trinidad (Lodge \& Pegler, 1990), creciendo sobre suelo. Esta colección se convierte en el primer registro para Bolivia.

Observaciones. Hygrocybe atrosquamosa Pegler pertenece al subgénero Hygrocybe (P.) P. Kumm. subsección Hygrocybe (P.) P. Kumm. y 
se caracteriza por presentar la superficie del píleo a veces fibrillosa y trama himenoforal paralela, a diferencia de la subsección Macrosporae R. Haller Aar. ex Bon. con píleo siempre fibrilloso y reacción oscura al tacto (Lodge et al., 2014). La subsección Hygrocybe incluye las especies $H$. atrosquamosa, H. astatogala (R. Heim) Heinem., H. conica (Schaeff.) P. Kumm. y sus variedades, H. nigrescens var. brevispora (Dennis) Pegler, $H$. singeri (A.H. Sm. \& Hesler) Singer, y $H$. olivaceonigra (P.D. Orton) M.M. Moser. (Lodge \& Pegler, 1990), presentando pseudocistidos en todos los casos. El material tipo referido a $H$. atrosquamosa presenta pseudocistidios de dimensiones $42-55 \times 5-11 \mu \mathrm{m}$; el material coleccionado en Bolivia posee pseudocistidios bien diferenciados del mismo rango de largo y más angostos $(30-55 \times 4-7 \mu \mathrm{m})$.

La especie morfológicamente más próxima a $H$. atrosquamosa es $H$. conica, presente en Bolivia (Melgarejo-Estrada et al., 2020b). Hygrocybe atrosquamosa difiriere de $H$. conica por presentar esporas más pequeñas (hasta $9 \mu \mathrm{m}$ de largo) ovoides a subglobosas, píleo a veces fibrilloso (Cantrell \& Lodge, 2000; Lodge \& Pegler, 1990), siempre umbonado y de colores rojos a anaranjados o grises (Lodge \& Pegler, 1990), pero nunca rojo intenso o escarlata como H. conica (Cardoso, 2017).

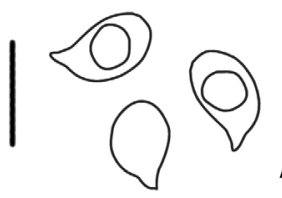

A
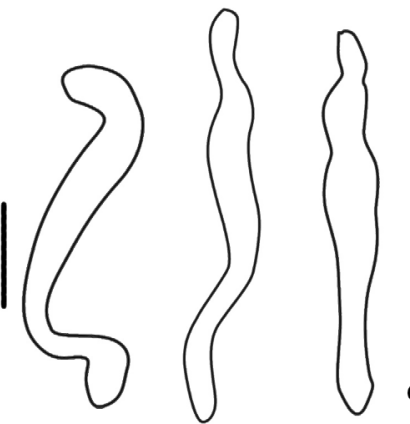

Fig. 5. Hygrocybe atrosquamosa. A, esporas. B, basidios. C, pseudocistidios. Escala $10 \mu \mathrm{m}$.
Otra especie cercana es $H$. astatogala, descrita para Madagascar y reportada para Australia (Young \& Wood, 1997), India (Farook et al., 2013) y el Neotrópico (Lodge \& Pegler, 1990). La principal diferencia con $H$. atrosquamosa, es el color rojo a violeta o castaño oscuro en $H$. astatogala; ambas desprenden un líquido translúcido al corte.

\section{Material examinado}

BOLIVIA. Cochabamba. Villa Tunari, comunidad de Paractito, hacia la entrada al Parque Nacional Carrasco, sobre suelo, 28-II-2019, E. MelgarejoEstrada \& M. Ibarra Merida 128 (BAFC 55395), $17^{\circ} 04^{\prime} 00^{\prime}$ ' S, 65' 29’00” O, 475 m s.m.

Mycena holoporphyra (Berk. \& Curt.) Singer, Sydowia 15: 64. 1962. Agaricus holoporphyrus Berk. \& Curt., Journal of the Linnean Society. Botany 10: 284 (1868). TIPO: Cuba, Wright 5 (Holotipo K, no visto). Figs. 6-7.

Píleo (Fig. 6) 20-23 mm de diám., cónicocampanulado, magenta oscuro (13E8) hacia el centro, magenta grisáceo (13B4) hacia el margen, estrías púrpura rojizas (14C8) y las zonas entre las estrías púrpura rosáceas (14A4) a púrpura rojizas (14B4), translúcidamente reticulado y estriado, carnoso, húmedo, glabro, margen sulcado. Laminillas adnatas a subdecurrentes, subdistantes, ventricosas, $2 \mathrm{~mm}$ de diám., púrpura-rosáceas (14A4) a púrpura claro (14A2) o blancas (14A1), lisas. Lamélulas de tercer orden, subdistantes, ventricosas, $2 \mathrm{~mm}$ de diám., púrpura claro (14A2) o blancas (14A1), lisas, con anastomosis entre laminillas. Pie 50-65 × 3-5 $\mathrm{mm}$, central, cilíndrico, recto, magenta grisáceo (13D5) aclarándose hacia la base a púrpura grisáceo (13B2), cartilaginoso, húmedo, hueco, glabro y velutinoso hacia la base. Anillo ausente. Olor y gusto rafanoide. Esporada blanca.

Esporas (Fig. 7A) 7-8 $\times 4-5 \mu \mathrm{m}, \mathrm{L}=6,29$, $\mathrm{A}=3,36, \mathrm{Q}=1,5-2,5, \mathrm{Qm}=1,92 \pm 0,3, \mathrm{~N}=15$, oblonga a elipsoidal, hialinas, lisas, pared delgada, amiloides, con contenido oleífero y/o gutular. Basidios (Fig. 7B) 18-30 × 5-6 $\mu \mathrm{m}$, 4-esporados, claviformes, esterigmas hasta $5 \mu \mathrm{m}$ de largo, pared delgada, hialinas. Pleurocistidios ausentes. 
Queilocistidios (Fig. 7C) (15-) 17-25 × 3-4 $\mu \mathrm{m}$, piriformes, a veces versiformes o rostrados, pared delgada, lisa, hialinos. Trama himenoforal regular, hifas 7-10 $\mu \mathrm{m}$ de diám., dextrinoides en Melzer. Pileipellis un cutis poco diferenciado, formado por hifas postradas, 2-4 $\mu \mathrm{m}$ de diám., pared lisa, delgada, hialina. Hipodermis formada por células vesiculosas y lisas. Fíbulas presentes. Estipitipellis un cutis similar a la pileipellis. Caulocistidios (Fig. 7D) (15-) 19-36 × 3-4 $\mu \mathrm{m}$, alargados, cilíndricos, a veces apretados hacia el ápice, pared apenas engrosada, lisa, hialina.

Distribución y hábitat. Gregario, sobre hojarasca. Mycena holoporphyra es una especie de amplia distribución tropical, reportada para Asia y África (Pegler, 1987), y para América en México, Costa Rica, Cuba, Trinidad, Martinica, Colombia, Brasil y Argentina (Niveiro et al., 2011). En los bosques nublados de las Yungas bolivianas crece sobre hojarasca. Esta colección se convierte en el primer registro para el país.

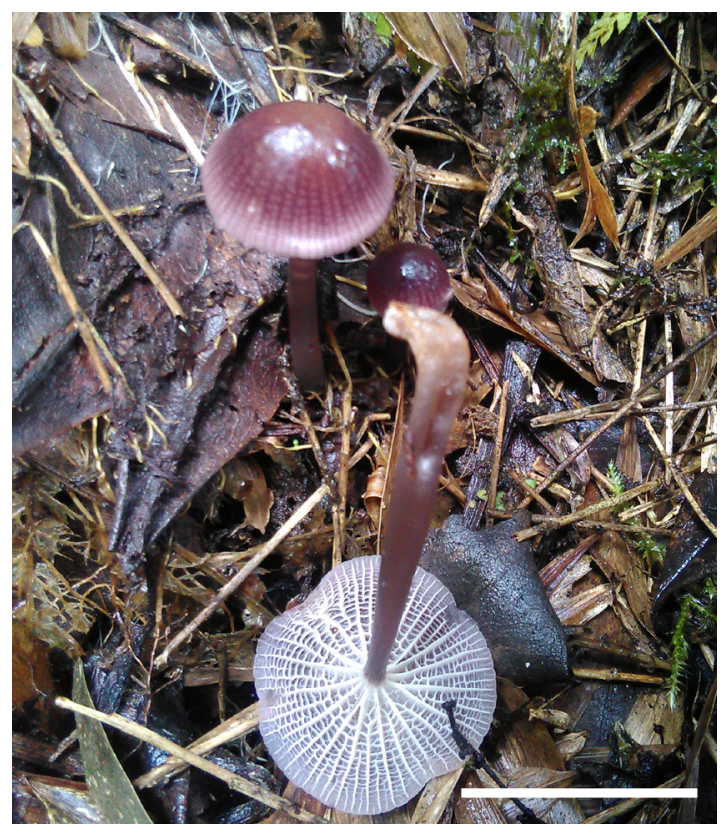

Fig. 6. Aspecto general y hábitat de Mycena holoporphyra (E. Melgarejo-Estrada \& M. Ibarra-Merida. 159; BAFC; E. Melgarejo-Estrada et al. 258, 335; BAFC, USZ). Escala: $2 \mathrm{~cm}$. Figura en color en la versión en línea http:// www.ojs.darwin.edu.ar/index.php/darwiniana/article/ view/890/1188

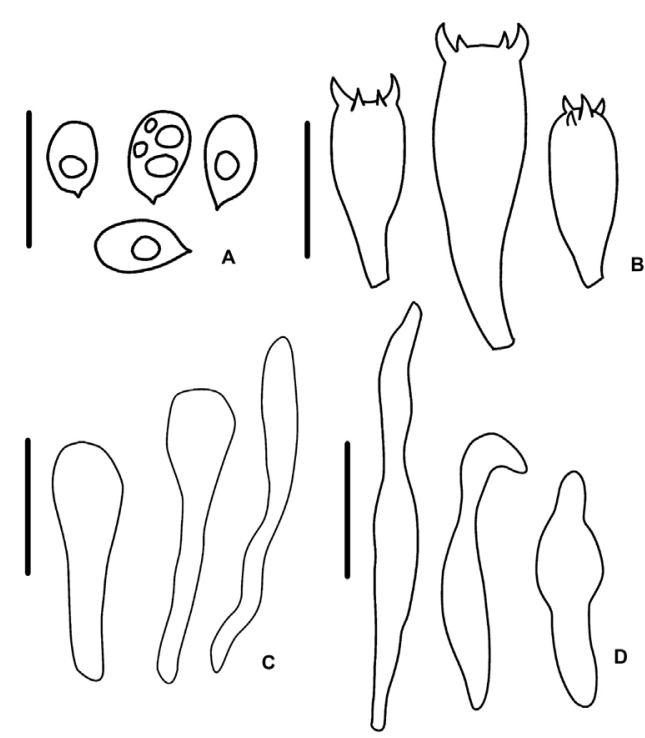

Fig. 7. Mycena holoporphyra. A, esporas. B, basidios. C, queilocistidios. D, caulocistidios. Escala: $10 \mu \mathrm{m}$.

Observaciones. Esta especie se caracteriza por poseer basidiomas completamente violáceos a púrpuras, olor rafanoide, esporas amiloides y ausencia de pleurocistidios (Niveiro et al., 2011). Si bien los márgenes de las laminillas son heteromorfos (Pegler, 1987), el material coleccionado en Bolivia difiere del material coleccionado en Argentina respecto al color de las laminillas: Niveiro et al. (2011) sugirieron colores vináceos, aclarándose hacia la arista de la laminilla, mientras que el material coleccionado en Bolivia posee tonos rosados a liláceos o blancos. Además, Zhishu et al. (1993) observaron un estípite velutinoso y Niveiro et al. (2011) mencionaron un tomento basal en el estípite; la muestra coleccionada en Bolivia posee un estípite velutinoso en la base, donde se observaron y midieron caulocistidios.

\section{Material examinado}

BOLIVIA. Cochabamba. Colomi, comunidad de Santa Isabel, Pampa Tambo, 2-III-2019, E. MelgarejoEstrada \& M. Ibarra Merida 159 (BAFC 53393), $17^{\circ}$ 11'37" S, 65 51' 55" O, 2406 m s.m. Santa Isabel, 10III-2019, $17^{\circ} 11^{\prime}$ 37” S, 65 51' 55" O, 2142 m s.m., E. Melgarejo-Estrada et al. 335 (USZ), Sehuencas, Parque Nacional Carrasco, bajando hacía el río Ivirizu, 30-X-2019, E. Melgarejo-Estrada et al. 258 (USZ), $17^{\circ} 30,28^{\prime} 00^{\prime \prime} \mathrm{S}, 65^{\circ} 16,72^{\prime} 00^{\prime \prime} \mathrm{O}, 2219$ m s.m. 


\section{AGRADECIMIENTOS}

A Rufford Foundation (26881-1) e Idea Wild Foundation por el financiamiento y equipo otorgado a la primera autora. Al Consejo Nacional de Investigaciones Científicas y Tecnológicas (CONICET), al Herbario del Oriente Boliviano (USZ) dependiente del Museo NKMUAGRM y al Herbario de Bolivia (LPB). A Andrea Romero por su colaboración y sus valiosos comentarios respecto a nomenclatura, a Andrés Coáguila, Bernardina Estrada, Maribel Ibarra y Mónica Cervantes por su apoyo en campo y a Mariana Valente y Oswaldo Maillard por la elaboración del mapa.

\section{BIBLIOGRAFÍA}

CABI Bioscience \& Landcare Research. Index fungorum. http:// www.indexfungorum.org/ [permanentemente actualizado, accedido: enero 2020].

Cabrera, A. L. \& A. Willink. 1980. Biogeografía de América Latina. Monografía 13. Washington, D.C: Serie de Biología.

Cantrell, S. A. \& D. J. Lodge. 2000. Hygrophoraceae (Agaricales) of the Greater Antilles. Hygrocybe subgenus Hygrocybe. Mycological Research 104: 873-878.

Cardoso, J. S. 2017. Hygrocybe sensu lato (Agaricales, Hygrophoraceae) na Mata Atlântica Brasileira. Tesis. Universidade Federal de Santa Catarina, Brasil.

Coimbra, V. R. M. 2015. Checklist of Central and South American Agaricales (Basidiomycota) II: Strophariaceae. Mycosphere 6(4): 441-458.

de Meijer, A. A. R. 2006. Preliminary list of the Macromycetes from the Brazilian state of Paraná. Boletim do Museu Botânico Municipal 68: 1-55.

Farook, V. A.; S. S. Khan \& P. Manimohan. 2013. A checklist of agarics (gilled mushrooms) of Kerala State, India. Mycosphere 4(1): 97-131.

Farr, M. L. \& J. A. Stevenson. 1963. Eine Erganzungsliste bolivianischer Pilze. Sydowia 17: 37-69.

Ibisch, P. \& G. Mérida. 2003. Biodiversidad: La riqueza de Bolivia. Estado de conocimiento y conservación. Santa Cruz de la Sierra: Fundación Amigos de la Naturaleza.

Ibisch, P. 1996. Neotropische Epiphytendiversität - das Beispiel Bolivien. Wiehl.: MartinaGalunder-Verlag.

Ibisch, P.; C. Nowicki \& R. Müller. 2001. El biocorredor Amboró-Madidi - primeros insumos botánicos para un Plan de Conservación. Revista de la Sociedad Boliviana de Botánica 3(1/2): 64-103.
Josse, C; F. Cuesta Camacho, G. Navarro, V. Barrena, M. T. Becerra, E. Cabrera, E. Chacón-Moreno, W. Ferreira, M. Peralvo, J. Saito, A. Tovar \& L. G. Naranjo. 2011. Geografía física y ecosistemas de los Andes, en: S. Herzog, R. Martinez, P. Jorgensen \& H. Tiessen (eds). Climate Change and biodiversity in the Tropical Andes. Inter-American Institute of Global Change Research (IAI) and Scientific Committee on Problems of the Environment (SCOPE), pp 117-180. Paris: SCOPE.

Kessler, M. \& S. G. Beck. 2001. Bolivia, en: M. Kappelle, A. Braun (eds.), Bosques nublados del Neotrópico, pp. 581622. Buenos Aires: Santo Domingo de Heredia.

Kornerup, A. \& J. H. Wanscher. 1978. Methuen Handbook of Colour. London: Eyre Methuen.

Largent, D. L. 1986. How to identify mushrooms to genus I: macroscopic features. Eureka: Mad River Press.

Lodge, D. J. \& D. N. Pegler. 1990. Hygrophoraceae of the Luquillo Mountains of Puerto Rico. Mycological Research 94(4): 443-456.

Lodge, J.; J. F. Ammirati, T. E. O'Dell, G. M. Mueller, S. M. Huhndorf, C. J. Wang, J. N. Stokland, J. P. Schmit, L. Ryvarden, P. R. Leacock, M. Mata, L. Umaña, Q. F. Wu \& D. Czederpiltz. 2004. Terrestrial and Lignicolous Macrofungi, en: G. M. Mueller, G. F. Bills, M. S. Foster (eds.), Biodiversity of Fungi, Inventory and Monitoring Methods, pp. 127-158. San Diego: Elsevier Academic Press.

Lodge, D. J.; M. Padamsee, P. B. Matheny, M. C. Aime, S. A. Cantrell, D. Boertmann, A. Kovalenko, A. Vizzini, B. T. M. Dentinger, P. M. Kirk, A. M. Ainsworth, J. M. Moncalvo, R. Vilgalys, E. Larsson, R. Lücking, G. W. Griffith, M. E. Smith, L. Norvell, D. E. Desjardin, S. A. Redhead, C. Ovrebo, E. B. Lickey, E. Ercole, K. W. Hughes, R. Courtecuisse, A. Young, M. Binder, A. M. Minnis, D. L. Lindner, B. OrtizSantana, J. Haight, T. Læssøe, T. J. Baroni, J. Geml \& T. Hattori. 2014. Molecular phylogeny, morphology, pigment chemistry and ecology in Hygrophoraceae (Agaricales). Fungal Diversity 64: 1-99.

Marín, C. 2018. Conceptos fundamentales en ecología de hongos del suelo: una propuesta pedagógica y de divulgación. Boletín de Micología. 33(1): 32-56.

Melgarejo, E. 2015. Algunos usos de los hongos silvestres de Bolivia en el contexto sudamericano. Kempffiana 11(1): 48-65.

Melgarejo-Estrada, E.; M. E. Suárez, D. Rocabado, O. Maillard \& B. E. Lechner. 2020a. Checklist of Bolivian Agaricales: 1. Species with dark and pink spore prints. Mycotaxon 134(4):739.

Melgarejo-Estrada, E.; D. Rocabado, M. E. Suárez, O. Maillard \& B. E. Lechner.2020b. Checklist of Bolivian Agaricales: 2. species with white and pale spore prints. Mycotaxon 135: 233. 


\section{E. MELGAREJO-ESTRADA ET AL. Nuevos registros de Agaricales para Bolivia}

Navarro, G. \& M. Maldonado. 2011. Geografia de Bolivia. Santa Cruz de la Sierra: Centro de Ecología Simón I. Patiño. Navarro, G. 2015. La vegetación de Bolivia. Santa Cruz de la Sierra: Centro de Ecología Simón I. Patiño.

Niveiro, N. \& E. Albertó. 2012. Checklist of the argentine Agaricales 3. Bolbitiaceae and Crepidotaceae. Mycotaxon 120: 505 .

Niveiro, N.; O. F. Popoff \& E. Albertó. 2011. Mycena holoporphyra (Mycenaceae, Agaricomycetes), primer registro para la Argentina. Darwiniana 49(2): 252-256.

Niveiro, N.; M. Uhart \& E. Albertó. 2020. Revision of the genera Agrocybe and Cyclocybe (Strophariaceae, Agaricales, Basidiomycota) in Argentina. Rodriguésia 71: e02272018.

Pegler, D.N. 1983. Agaric flora of Lesser Antilles. Kew Bulletin Additional Series 9: 1-668.

Pegler, D. N. 1987. A revision of the Agaricales of Cuba 1. Species described by Berkeley \& Curtis. Kew Bulletin 42: 501-585.

Piepenbring, M. 2003. Fungi, en: P. Ibisch \& G. Mérida (eds). Biodiversidad: La riqueza de Bolivia. Estado de conocimiento y conservación, pp 90-96. Santa Cruz de la Sierra: FAN.

Piepenbring, M. 2015. Introducción a la Micología en los Trópicos. Estados Unidos: The American Phytopathological Society.

Rick, J. 1907. Contributio ad monographiam Agaricacearum et Polyporacearum Brasiliensium. Brotéria, série. botanica 6: 65-92.

Rick, J. 1938. Agarici Riograndenses. Lilloa 2: 251-16.

Rocabado, D.; J. E. Wright, O. Maillard \& N. F. Munchenik. 2007. Catálogo de los Gasteromycetes (Fungi: Basidiomycotina) de Bolivia. Kempffiana 3(1): 3-13.

SERNAP (Servicio Nacional de Áreas Protegidas-Bolivia), Ministerio de Medio Ambiente y Agua http://sernap.gob. bo/page/46/?paged $=46 \quad$ [permanentemente actualizado, accedido: febrero 2020].

Singer, R. 1953. Type studies on Basidiomycetes. VI. Lilloa 26: 57-159.

Singer, R. 1986. The Agaricales in Modern Taxonomy. Koenigstein: Koeltz Scientific Books.
Singer, R. \& A. P. L. Digilio. 1952 (1951). Pródromo de la Flora Agaricina Argentina. Lilloa 25: 6-461.

Spegazzini, C. 1899 (1898). Fungi argentini novi vel critici. Anales Museo Nacional de Historia Natural de Buenos Aires 6: 6-365.

Stevenson, J. A. \& M. Cárdenas. 1949. Lista preliminar de los hongos de Bolivia. Lilloa 21: 77-134.

Thiers, B. 2020. Index Herbariorum: a global directory of public herbaria and associated staff. New York Botanical Garden's Virtual Herbarium, http://sweetgum.nybg.org/ih [permanentemente actualizado, accedido: enero 2020].

Turland, N. J., J. H. Wiersema, F. R. Barrie, W. Greuter, D. L. Hawksworth, P. S. Herendeen, S. Knapp, W-H. Kusber, D-Z. Li, K. Marhold, T. W. May, J. McNeill, A. M. Monro, J. Prado, M. J. Price \& G. F. Smith (eds.). 2018. International Code of Nomenclature for algae, fungi, and plants (Shenzhen Code): adopted by the Nineteenth International Botanical Congress Shenzhen, China, July 2017. Regnum Vegetabile 159. Glashütten: Koeltz Botanical Books.

Uhart, M. 2006. Biodiversidad del género Agrocybe (Basidiomycetes, Agaricales) en la República Argentina. Estudios de filogenia molecular, compatibilidad sexual y cultivo intensivo del complejo Agrocybe cylindrica. Tesis. Universidad Nacional de General San Martín, San Martín, Prov. Buenos Aires.

Watling, R. 1992. Observation on the Bolbitiaceae 30. Some brazilian taxa. Boletín de la Sociedad Argentina de Botánica 28(1-4): 77-103.

Wright, J. E. \& E. Albertó. 2002. Guía de hongos de la Región Pampeana. I. Hongos con laminillas. Buenos Aires: L.O.L.A.

Young, A. M. \& A. E. Wood. 1997. Studies on the Hygrophoraceae (Fungi, Homobasidiomycetes, Agaricales) of Australia. Australian Systematic Botany 10: 911-1030.

Young, B.E. 2007. Distribución de las especies endémicas en la vertiente oriental de los Andes en Perú y Bolivia. Virginia: NatureServe.

Zhishu, B., Z. Guoyang \& L. Taihui. 1993. The Macrofungos Flora of China's Guangdong Province. Hong Kong: The Chinese University Press. 